\title{
Wavelet Packets and De-noising Based on Higher-Order-Statistics for transient Detection
}

\author{
Philippe Ravier* and Pierre-Olivier Amblard** \\ ${ }^{*}$ Laboratoire d'Électronique, Signaux, Images LESI-ESPEO \\ BP 6744-45067 Orléans Cédex 2-France \\ e-mail: Philippe.Ravier@univ-orleans.fr \\ Tel: (33)-2-38-49-48-63 \\ Fax: (33)-2-38-41-72-45
}

** Laboratoire des Images et des Signaux LIS-ENSIEG

UMR 5083 Groupe Non Linéaire

BP46 - 38402 St-Martin d'Hères Cédex - France

e-mail: Bidou.Amblard@lis.inpg.fr

Tel: (33)-4-76-82-71-06

Fax: (33)-4-76-82-63-84 
In this paper, we present a detector of transient acoustic signals that combines two powerful detection tools: A local wavelet analysis and higher-order statistical properties of the signals. Using both techniques makes detection possible in low signal-to-noise ratio conditions, when other means of detection are no longer sufficient. The proposed algorithm uses the adapted wavelet packet transform. It leads to a partition of the signal which is 'optimal' according to a criterion that tests the Gaussian nature of the frequency bands. To get a time dependent detection curve, we perform a de-noising procedure on the wavelet coefficients: The Gaussian coefficients are set to zero. We then apply a classical method of detection on the time reconstructed denoised signal. We study the performance of the detector in terms of experimental ROC curves. We show that the detector performs better than decompositions using other classical splitting criteria. In a last part, we present an application of the algorithm on real flow recordings of nuclear plant pipings. The detector indicates the presence of a missing body in the piping at some instants not seen with a classical energy detector.

Nous présentons dans cet article un nouveau détecteur de signaux transitoires acoustiques. Ce détecteur tire parti de deux techniques puissantes utilisées en détection : une analyse locale par ondelettes combinée à une exploitation des propriétés statistiques aux ordres supérieurs des signaux. Cette approche rend possible la détection dans des conditions de rapport signal sur bruit difficiles alors que les méthodes classiques demeurent insuffisantes. L'algorithme proposé utilise les paquets d'ondelettes qui réalisent un pavage du plan temps-fréquence adapté à l'observation. Il aboutit à la construction d'une base de décomposition qui est "optimale" selon un critère qui teste la nature gaussienne des bandes fréquentielles. Pour obtenir une courbe de détection temporelle, on effectue un débruitage du signal par mise à zéro des coefficients de bandes gaussiennes. Une méthode classique de détection est ensuite appliquée sur le signal débruité reconstruit en temps.

On étudie les performances du détecteur en terme de courbes COR expérimentales. Nous montrons que le détecteur donne de meilleurs résultats que les décompositions qui utilisent d'autres critères classiques de segmentation. Dans une dernière partie, une application de l'algorithme à un enregistrement de circulation hydraulique dans une centrale nucléaire est proposée. Le détecteur indique la présence d'un corps errant à des instants non révélés par un détecteur classique d'énergie.

Key words: transient detection, wavelet packets, multiresolution analysis, adapted segmentation, de-noising, higher-order statistics, ROC performance curves.

\section{Introduction}

\subsection{Presentation of the problem}

Wavelets and Higher-Order-Statistics are two of the most successful advances in the field of signal processing of the twenty last years. Both techniques are powerful tools that can efficiently be used for detection applications. But rare are the applications where researchers simultaneously have tried to take advantage of the two approaches by combining them. We propose to do so in this paper and show how such a combination can improve the quality of detection.

The problem addressed here concerns the detection of short time impulsive signals that we call 
transient signals. The detection of this kind of signal has many applications. In biological domains, the detection and exact localization of transients in ECG or EEG recordings is of great importance $[18,19,5]$. In $[1]$, the authors detect wide-band transients that are pressure drop signals originating from a developed turbulence experiment. In passive sonar problems, the detection of transient acoustic signals constitutes an alternative to other classical sonar detection means because of the progress realized in reducing acoustic noise generated by naval vessels. Thus the traditional means of detection are insufficiently reliable $[6,17]$.

In the problem addressed here, the signals are supposed to be embedded in a sufficiently strong additive noise so that phenomena cannot be visually detected considering the observed temporal shape. The detector proposed aims to perform better than any energy detector so that false alarm or missed detection situations are mitigated. In addition to severe signal-to-noise ratio contexts, the signals of interest suffer from a lack of information: This makes the problem very difficult to solve. The signals to detect are poorly defined for the following reasons:

- Their temporal signal shapes are not exactly known,

- Their arrival times are not known and generally need to be estimated,

- Their brief existence bring reduced information for their characterization.

However, we need to define the objects more precisely. Many of them have an oscillatory shape yielding high peaks in their spectrum. This is especially true for the signals we considered such as transients generated by metallic shocks, cracks or slams.

Transient signals can therefore be described as models with various parameters. Friedlander and Porat in [7] have proposed a theoretical solution of the problem assuming the signal was composed of damped parameterized sines corrupted by a white Gaussian noise. Detectors investigated are based on a class of linear data transforms. Actually, models are rather far from representing all the variety of transient signals. So we intentionally chose a non parametric approach to prevent from restricting the problem to a class of particular signals. Transients will then be characterized through their statistical properties which contrast with the properties of the noise.

We discuss in the next section how we already proposed a detector based on Malvar wavelets and its working limitations for assumed white Gaussian stationary noise. Wavelet packets are proposed instead in more realistic situations when the noise is colored and stationary.

\subsection{From Malvar wavelets to wavelet packets}

In a previous work, we have shown that Malvar wavelets can successfully be used when the noise is stationary white and Gaussian [16]. The basic idea consists in discriminating the noise from the transient signal by segmenting the observation in fine temporal slices when a transient is present and in large ones when there is noise only. The purpose is to get the 'best' segmentation which is adapted to a desired criterion. Actually, the segmentation corresponds to selecting a basis among the set of all possible bases which is related to a corresponding partition in the time-frequency plane.

The criterion to choose the best basis is based on the Gaussianity coefficients: When two adjacent segments have Gaussian coefficients, they are merged, otherwise they are kept separated. Determination of the Gaussianity of the wavelet coefficients is done using Higher-Order Statistics. When the 
noise is white stationary, the coefficients remain white stationary so that statistics can be estimated by replacing mathematical expectations with average computing. When the noise is colored, wavelet coefficients in the same segment cannot be considered stationary so that the estimation is not correct. For that reason, the 'split and merge temporal slices' criterion that is used for the best basis search, fails to give good results. To circumvent this problem, we propose to work in the frequency domain using wavelet packets. The best basis is chosen in the same way: Merging 'Gaussian frequency bands'. In this case, the estimation process is correct because the coefficients in the same frequency band are stationary. This point is described further in the paper.

The procedure used to search for the best basis is schematically described in the figure 1 for the two cases described below. In the first case depicted in [16], the best basis is initialized with a set of Malvar wavelets supported on minimal equally temporal slices. The merging criterion is applied on pairs of adjacent slices. It leads to the merging of the slices according to the Gaussianity of the wavelet coefficients in each slice. When merging, considered wavelets are replaced by the same number of twice longer supported wavelets. In the example of figure 1, the third slice is supposed to have nonGaussian coefficients. The first step merges the slices 1-2, 5-6 and 7-8. In a second step, the procedure leads to the merging of the last two slices obtained in the first step. The best basis is reached.

In the second case (figure 1b), the process is exactly the same except the signal is initially segmented in equal frequency bands. The merging criterion is applied to adjacent frequency bands. The time-frequency plane evolves according to the frequency variable which is "active" in the tiling. Inversely, the time variable passively reacts because frequency segmentation imposes the tiling in the temporal domain.

Considering the detection problem, the purpose, however, is to get a time dependent detection statistic. The obtained time frequency plane tiling actually corresponds to a set of admissible wavelets constituting a basis. The signal is projected on each element of this basis producing decomposition coefficients. To return to a single temporal curve, a de-noising procedure setting Gaussian coefficients to zero is performed. A denoised signal is reconstructed from the retained coefficients. After that, a standard detection algorithm is applied.

The exact procedure of detection is detailed in section 3. Studies of performance are described.

The method is illustrated in section 4 with the detection of a screw bolt that has been forgotten in nuclear plant pipings.

In the section hereafter, we briefly introduce wavelet packets defined as an extension of the multiresolution analysis principles. We want to explain their construction as well as the coefficient computation. Before that, we give a justification in the use of adapted wavelets for the problem of transient detection. 


\section{Using Wavelet Packets for detection}

\subsection{Interest of adapted wavelet transforms}

In a detection problem, time-frequency representations are used to distribute the energy of a signal on the time-frequency plane, in such a way that relevant information can be extracted to make a good detection. The results generally depend on the retained parameters and on the method itself used as a time-frequency representation.

For example, discrete windowed Fourier 'bases' tile the time-frequency plane in regular cells which all have the same uncertainties. Discrete wavelet bases tile the time-frequency plane more naturally, according to the evolution velocity of phenomena. A low frequency needs to be observed on a long time to be correctly estimated whereas a high frequency can rapidly change at any time. Therefore, timefrequency localization naturally depends on the 'observation scale'. However, these decompositions impose a fixed tiling shape in the time-frequency plane, independent of the observation. Moreover, choosing the analysis window size or the decomposition depth is more or less done empirically. On the other hand, it is possible, using adapted wavelet transforms, to obtain adapted tilings in the time-frequency plane, automatically according to the observation.

In a detection problem, the idea is to adapt the time-frequency tiling to the signature of the signals of interest. Considering figure 2, the pattern represented on the left corresponds to a transient signal in a continuous short time Fourier representation. An adapted wavelet transform using wavelet packets as depicted in panel (c) allows the time-frequency pattern to be insulated with an appropriate tiling. In practice, the signal is of course strongly polluted and seeking for an adapted wavelet transform will perform a better detection, if achievable.

The key point is to obtain a tiling showing a 'pre-detection' of the events. A time-frequency tiling is associated to a retained wavelet basis among all the possible wavelet decomposition bases. Basically, a basis is constituted by some admissible elements in a set of wavelet packets. Wavelet packets and their properties are now presented.

\subsection{Definitions and properties}

We introduce wavelet packets as an extension of the multiresolution analysis (MRA). This mathematical formalism has been developed for fast computation of the discrete wavelet transform coefficients [14]. The MRA is built on two time series filters $h_{n}$ and $g_{n}$. The construction of wavelet families as well as the coefficient computation rely on these single sequences.

From $h_{n}$ and $g_{n}$, we need to define the two operators $\mathcal{H}$ and $\mathcal{G}$ as filters on the digital signal $x_{n}$ :

$$
\begin{aligned}
(\mathcal{H} x)_{n} & =\sum_{k} h_{k} x_{n-k} \\
(\mathcal{G} x)_{n} & =\sum_{k} g_{k} x_{n-k} .
\end{aligned}
$$


When $x$ is a function of the continuous variable $t$, we define:

$$
\begin{aligned}
(\mathcal{H} x)(t) & =\sum_{k} h_{k} x(t-k) \\
(\mathcal{G} x)(t) & =\sum_{k} g_{k} x(t-k) .
\end{aligned}
$$

With these conventions, the classical scale and wavelet functions read:

$$
\begin{aligned}
\phi & =\sigma \mathcal{H} \phi \\
\psi & =\sigma \mathcal{G} \phi .
\end{aligned}
$$

where $\sigma$ is a compression operator applied on a continuous function $f(t)$ such as: $\sigma f(t)=\sqrt{2} f(2 t)$. Wavelet packets are created by further applying the operators either on $\phi$ or on $\psi$. It produces the infinity $\psi_{0}, \psi_{1}, \psi_{2}, \ldots$ of wavelets indexed by the arrangement $f \in \mathbb{N}$ defined as:

$$
\begin{aligned}
\psi_{2 f} & =\sigma \mathcal{H} \psi_{f} \\
\psi_{2 f+1} & =\sigma \mathcal{G} \psi_{f} \quad \text { with } \quad \psi_{0}=\sigma \mathcal{H} \psi_{0} .
\end{aligned}
$$

Note that $\psi_{0}=\phi$ and $\psi_{1}=\psi$. The family $\left\{\psi_{f}(t-p), p \in \mathbb{Z}, f \in \mathbb{N}\right\}$ constitutes an orthonormal basis of $L^{2}(\mathbb{R})[20]$ (The sequences $\left\{h_{n}\right\}$ and $\left\{g_{n}\right\}$ are called quadrature mirror filters).

In the frequency domain, the family $\left\{\widehat{\psi_{f}}\right\}$, where $\widehat{\psi_{f}}(\nu)$ stands for the Fourier transform of $\psi_{f}(t)$, acts as a collection of subband filters (see figure $3 \mathrm{~b}$ for $f=4$ ).

We now shift and scale the $\psi_{f}(t)$ to obtain multiscale wavelet packets:

$$
\psi_{s, f, p}(t)=2^{-\frac{s}{2}} \psi_{f}\left(2^{-s} t-p\right), \quad s, p \in \mathbb{Z}, f \in \mathbb{N}
$$

The operators $\mathcal{H}$ and $\mathcal{G}$ can again be used to express wavelet packets from one scale to the following one. We note the decimation operator as $(\downarrow x)_{n}=x_{2 n}$ and $\mathcal{H}^{\triangleleft}$ the operator $\mathcal{H}$ for the inverse sequence $h_{-n}$. The recursive expressions are derived:

$$
\begin{aligned}
\psi_{s+1,2 f, p} & =\left(\downarrow \mathcal{H}^{\triangleleft} \psi_{s, f, .}\right)_{p} \\
\psi_{s+1,2 f+1, p} & =\left(\downarrow \mathcal{G}^{\triangleleft} \psi_{s, f, .}\right)_{p} .
\end{aligned}
$$

Note that incrementing the scale modifies the 'frequency index' $f$ because increasing the scale corresponds to a finer frequency analysis and authorizes a greater number of frequencies. In the time/frequency plane, applying $\downarrow \mathcal{H}^{\triangleleft}$ and $\downarrow \mathcal{G}^{\triangleleft}$ 'splits' the initial band into two subbands which doubles the frequency resolution as illustrated in figure 4 . The inverse 'merging' operator merges two adjacent frequency bands in a single larger one (figure 5).

In this kind of diagram, the entire time-frequency cell information is supposed to be carried by the wavelet coefficient, which is the inner product of $\psi_{s, f, p}(t)$ with the signal $x(t)$ :

$$
W P_{s, f, p}=\left\langle x, \psi_{s, f, p}\right\rangle \quad \text { in the } L^{2}(\mathbb{R}) \text { sense. }
$$

Remarkably, the wavelet coefficients can be recursively evaluated from the scale $s$ to the next one 
$s+1$ in the same way it can be done with the wavelet functions:

$$
\begin{aligned}
W P_{s+1,2 f, p} & =\left(\downarrow \mathcal{H}^{\triangleleft} W P_{s, f, .}\right)_{p} \\
W P_{s+1,2 f+1, p} & =\left(\downarrow \mathcal{G}^{\triangleleft} W P_{s, f, .}\right)_{p} .
\end{aligned}
$$

In the merging process, coefficients are reconstructed by the following relation:

$$
W P_{s, f, p}=\left(\mathcal{H} \uparrow W P_{s+1,2 f, .}\right)_{p}+\left(\mathcal{G} \uparrow W P_{s+1,2 f+1, .}\right)_{p}
$$

where the interpolation operator acts like $(\uparrow x)_{2 n}=x_{n}$ and $(\uparrow x)_{2 n+1}=0$. Since wavelets are orthonormal, it is possible to easily reconstruct a signal from the coefficients and to obtain filtered versions of the original signal at frequency $f$ and scale $s$, such that $x_{s f}(t)=\sum_{p} W P_{s, f, p} \psi_{s, f, p}(t)$.

A wavelet packet basis of $L^{2}(\mathbb{R})$ can be constructed by appropriately selecting a set of wavelets by their triplets $(s, f, p)$ among the whole collection $\left\{\psi_{s, f, p}(t), s, p \in \mathbb{Z}\right.$ and $\left.f \in \mathbb{N}\right\}$. The indices $s, f$

must be selected such that dyadic disjoint intervals $I_{s f}=\left[\frac{f}{2^{s}}, \frac{f+1}{2^{s}}\right] f \in \mathbb{Z}, s \in \mathbb{Z}$ cover the entire real positive frequency axis. Then the functions $\psi_{f}\left(2^{-s} t-p\right), p \in \mathbb{Z}$ such that $\cup_{s, f} I_{s f}=\mathbb{R}^{+}$constitute an orthonormal basis of $L^{2}(\mathbb{R})$. Let us denote $\mathcal{B}$ all the possible constructible bases derived from the admissible sets in $\mathcal{T}=\left\{(s, f, p) / \cup_{s, f}\left[2^{-s} f, 2^{-s}(f+1)\right]=\mathbb{R}^{+}, p \in \mathbb{Z}\right\}$. Then the signal $x(t)$ reads:

$$
x(t)=\sum_{(s, f, p) \in \mathcal{T}} W P_{s, f, p} \psi_{s, f, p}(t) .
$$

This relation not only stands for an expansion of the signal on the $\left\{\psi_{s, f, p}(t),(s, f, p) \in \mathcal{T}\right\}$ family but also expresses a signal reconstruction from a set of wavelet coefficients.

Practically, for an $N=2^{L}$ points digital signal $x_{n}$, the number of coefficients at a given frequency band is scale dependent. At a scale depth $s$, we have $f=0, \ldots, 2^{s}-1$ frequency indices and $p=$ $0, \ldots, 2^{L-s}$ cell positions equally distributed on the time axis. Globally, the number of coefficients in the time-frequency plane is always the same (i.e. $N$ the sample number of the original signal) whatever the adapted tiling considered.

We now explain how to obtain the best adapted wavelet basis.

\subsection{Towards the 'best' adapted wavelet basis}

The key point in the search for the best basis results in the ability of making a basis evolve towards the 'best' one by iteratively substituting elements of the basis with other admissible elements. For example, the $\left\{\psi_{s, f}(p), p=0 . .2^{L-s}\right\}$ wavelets can be substituted by the functions $\left\{\psi_{s+1,2 f+\epsilon}(p), \epsilon=\right.$ 0,1 and $\left.p=0 . . \frac{2^{L-s}}{2}\right\}$. In the manipulation, the number of wavelets is unchanged for the basis.

This operation affects the time-frequency tiling by splitting or merging frequency bands when changing the scale parameter. The decision of modifying or not the initial basis at each step is taken according to a criterion. Practically, we need to start with a given initial basis. The basis is composed of a set of wavelets with the same largest given scale. In the time-frequency plane, it corresponds to slicing the Nyquist band in equal frequency bands with the desired finest width. The split and merge principle is only applied in the merge sense and selects the frequency bands to be merged, from the finest ones to the largest ones. 
The 'split and merge' algorithm compares a function evaluated on the coefficients of two adjacent frequency bands. Traditional functions such as Shannon entropy, concentration in $l^{p}$ or logarithm of energy are used. These functions stand for an information measure and the aim is to construct a basis with the minimum cost, i.e. to minimize the total information measure.

Let us call $\mathcal{M}$ an information cost functional computed on the wavelet packet coefficients:

$$
\mathcal{M}\left\{W P_{s, f, p}\right\}=\sum_{(s, f) \in \mathcal{B}} \mathcal{I}\left\{\left|W P_{s, f}(p)\right|\right\}
$$

for a chosen $\{(s, f, p)\}$ family. Here $\mathcal{I}$ is a real valued additive information measure. The best basis is obtained by minimizing $\mathcal{M}\{W P\}$. This corresponds to choosing the appropriate sequence of $(s, f, p)$ indices in $\mathcal{B}$ necessary to cover the time-frequency plane. The minimization is made recursively.

Considering two sequences of coefficients $\left\{W P_{s, 2 f}(p)\right\}$ and $\left\{W P_{s, 2 f+1}(p)\right\}$ at a fixed scale $s$ and frequency $f$, and the sequence covering the same frequency band at scale $s-1\left\{W P_{s-1, f}(p)\right\}$, the criterion must decide which coefficient sets must be kept for the 'best' decomposition. The choice of minimum information cost is generally considered which provides the following decision criterion:

$$
\left\{\begin{array}{l}
\text { If } \mathcal{I}\left\{W P_{s, 2 f}(p)\right\}+\mathcal{I}\left\{W P_{s, 2 f+1}(p)\right\}<\mathcal{I}\left\{W P_{s-1, f}(p)\right\} \\
\text { then keep the coefficients } W P_{s, 2 f}(p) \text { and } W P_{s, 2 f+1}(p) \text { for the wavelet decomposition } \\
\text { Otherwise replace the coefficients } W P_{s, 2 f}(p) \text { and } W P_{s, 2 f+1}(p) \text { by the coefficients } W P_{s-1, f}(p) .
\end{array}\right.
$$

For each decision, the minimum cost must be kept.

This decision criterion is built under the idea of coding or compression applications, where the information must be carried by the minimum number of coefficients. Remember that we want to detect transient signals which is not necessarily compatible with a construction in a minimum cost sense. The purpose in the case of detection is quite different: The criterion must realize the segmentation that makes the desired signals clearly appear in the time-frequency representation.

The next part describes the criterion we propose for that purpose.

\section{Procedure of detection and performance}

The procedure of detection is based on a best basis search procedure which is able to increase the signal to noise ratio by an appropriate time frequency tiling.

\subsection{Searching for the best basis and de-noising}

The noise is supposed to be stationary. Transients are often oscillating producing some large coefficients in a wavelet decomposition. Therefore, the amplitudes of the coefficients are rather far from being smoothly distributed. By contrast, the noise samples are Gaussian distributed when observed for a sufficiently long time [3]. The discrimination between transients and the noise can therefore be created using a Gaussianity measure. The presence of a transient will generate nonGaussian 
coefficients at some frequency bands where the transient exists. On the other hand, Gaussian coefficients will represent noise only cases. Thus a natural idea consists in merging the frequency bands which have the same Gaussian nature. Finally, the procedure 'merge frequency bands when they are Gaussian' reveals transients by showing a fine frequency segmentation for nonGaussian events.

The problem now is to get a Gaussianity measure. Higher Order Statistics are traditionally used to accomplish this aim. We must consider a normalized measure because the Gaussianity measure must not depend on the signal energy at each frequency band. We want to solve the problem of detection for stationary colored noise. A good candidate is the kurtosis which is the normalized version of the fourth order cumulant [13]. Gaussian process theoretically have a kurtosis value that equals zero.

One could have chosen a functional of various Gaussianity measures. For example, using both skewness and kurtosis permits one to take into account the asymmetry and the tail behavior of the probability density function of the coefficients. However in this work, we have only kept a kurtosis estimator to measure the Gaussianity.

Theoretically, measuring the Gaussianity for a set of coefficients consists of comparing the kurtosis value with zero. In practice, the kurtosis is estimated and its value is authorized to exist in a confidence interval which is conditioned by the probability properties of the estimator. An asymptotic regime could lead to a probability density function estimator, but experimental conditions are not asymptotic. So we need to frame the estimator by another way, for example using the BienayméTchebychev inequality. Given a desired confidence percentage, the estimator can be framed between two values depending on the first statistics of the estimator. In the case where the $N$ coefficients $W P_{s, f, p}$ are white and Gaussian, bias and variance of the kurtosis estimator $\widehat{k_{4}}$ are evaluated as

$$
\left\{\begin{aligned}
B\left(\widehat{k_{4}}\right) & =-6 / N \\
\operatorname{Var}\left(\widehat{k_{4}}\right) & =24 / N
\end{aligned}\right.
$$

when the kurtosis is classically computed by a statistical estimation of the mean as

$$
\widehat{k_{4}}\left(W P_{s, f}\right)=\frac{E\left[W P_{s, f}^{4}\right]}{\left(E\left[W P_{s, f}^{2}\right]\right)^{2}}-3=N \frac{\sum_{p=1}^{N} W P_{s, f}(p)^{4}}{\left(\sum_{p=1}^{N} W P_{s, f}(p)^{2}\right)^{2}}-3
$$

The Bienaymé-Tchebychev inequality allows a Gaussian estimator to move between $-\sqrt{24 / N} / \sqrt{1-\alpha}+$ $6 / N$ and $\sqrt{24 / N} / \sqrt{1-\alpha}+6 / N$ with an $\alpha$ authorized confidence percentage value. This condition uses the first estimator statistics of the kurtosis which have been analytically evaluated when the samples are white and Gaussian. In the present case, the signal samples are Gaussian and correlated. The wavelet coefficients clearly stay Gaussian when applying the linear wavelet transform. Moreover, wavelets and wavelet packets can be considered as good decorrelators, if the number of vanishing moments of the mother wavelet is sufficiently high. Assuming this condition is realized, the coefficients can be considered as nearly white, which justifies the application of the Bienaymé-Tchebychev inequality given above. More precisely, the observed decorrelation process is not the same for all the wavelet coefficients. Actually, the second order coefficient moments which are a measure of the whiteness are linked to the frequency localization of the wavelet packets, also depending on the $\left\{h_{n}, g_{n}\right\}$ sequences used $([15])$.

Note that an unbiased version of the kurtosis estimator can be derived using $k$-statistics [12]: The kurtosis is calculated using unbiased estimations of the cumulants of order 2 and 4 . Its variance is 
given by

$$
\operatorname{Var}\left(\widehat{k}_{4}^{\text {unbiased }}\right)=\frac{24 N(N-1)^{2}}{(N-3)(N-2)(N+3)(N+5)}
$$

for an $N$ samples sequence. Choosing or not a biased estimator has a minor influence on the performances in the present case. The simple test $\left|\widehat{k_{4}}\right|<\sqrt{24 / N} / \sqrt{1-\alpha}$ is retained for the Gaussianity measure.

In practice, the best basis search strategy consists of choosing a maximal wavelet decomposition depth of the signal. This maximal depth nlevel defines the finest bandwidth available for the decomposition as $2^{\text {nlevel-1 }} * f_{s}$, where $f_{s}$ stands for the frequency sampling. An initial basis composed of the wavelets at the greatest resolution level is arbitrarily selected. At this level, frequency bands are merged or not according to the diagnosis given by the Bienaymé-Tchebychev inequality. It means that two components of the basis $\psi_{s, 2 f, p}$ and $\psi_{s, 2 f+1, p}$ can be replaced by the admissible wavelet $\psi_{s-1, f, p}$. The merging test is repeated at each resolution level $s$ on adjacent pairs indexed by $2 f$ and $2 f+1$ with $f \in\left[0 ; 2^{s-1}-1\right]$, up to the root corresponding to the entire temporal signal. The procedure leads to the selection of the triplets $\left\{(s, f, p)\right.$ such that $\left.(s, f, p) \in B^{*}\right\}$ where $B^{*}$ stands for the searched 'best' basis in all the admissible bases of $B$.

In the operation, the contrast between Gaussian and nonGaussian regions is enhanced in the time-frequency representation. For the purpose of detection, the idea is to keep only nonGaussian coefficients which allows to get rid of the greatest part of the disturbing noise. This classical technique is called de-noising and consists in setting to zero all the wavelet coefficients whose magnitude is below an appropriate threshold. The method used here is quite different in so far as the whole coefficients in the same frequency band are set to zero or not according to the Gaussianity property. We have proposed to measure the Gaussianity with the kurtosis of the coefficients at each frequency band. So in a de-noising procedure, all the coefficients representing the decomposition in a same frequency band are set to zero or not according to the Gaussianity of the band.

How can the de-noising threshold be chosen ? Only the finest frequency bands actually reveal local nonGaussianities. The other larger bands are not relevant for making a decision on the Gaussianity nature because of the constraining dyadic structure of the decomposition. The de-noising threshold is naturally set as the Byenaymé-Tchebychev bound evaluated for the finest frequency band.

For an $N=2^{L}$ samples observed signal $x$, the threshold therefore reads:

$$
\eta=\frac{1}{\sqrt{1-\alpha \%}} \cdot \sqrt{\frac{24}{N_{\min }}} \quad \text { where } \quad N_{\min }=2^{L-\text { nlevel }} .
$$

The threshold depends on the number of points at the finest frequency band which is in relation to the number of decomposition levels nlevel.

Other kinds of thresholding techniques exist. They correspond to different strategies which are not appropriate for the problem discussed in this paper. Here we operate in the sense of detection. It consists of differentiating Gaussian and nonGaussian areas and this strategy leads to an adapted thresholding method with a natural thresholding value.

A validation of this approach is given in the paragraph 3.3, with a set of comparison and performance tests. An illustration on real signals is proposed in the next paragraph. The detection 
procedure is more precisely described.

\subsection{Illustration with real signals}

The two components signal + noise have been recorded independently. This allows to generate noisy realizations of the transient with a desired signal-to-noise ratio.

We have retained a set of four transient signals stemming from real experiments in underwater acoustics. These signals depicted in figure 6 have in common a brief existence and present one or several narrow bands. The peaks characterize oscillatory responses of the material or structure after a shock given to generate transient signals (See [16] for a precise description of their generation). As these signals look rather complex in the frequency domain, the signal-to-noise ratio has been defined as the energy ratio on the support of the transient, as explained in [2].

The noise has been directly recorded in the Mediterranean sea at a $16 \mathrm{kHz}$ sampling frequency. The noise is colored with high energy in the low frequencies, presenting a $-6 \mathrm{~dB} /$ octave spectral decrease up to about $1500 \mathrm{~Hz}$ (figure 7). The kurtosis estimated beyond 20000 points is less than 0.05 .

We have generated a realization of the transient 'manhole cover shock' embedded in the sea noise at $-6 \mathrm{~dB}$. The detection scheme we propose is illustrated with the panels of figure 8 .

The procedure of detection is the following, assuming the parameters have been fixed (decomposition depth nlevel, confidence percentage $\alpha$, wavelet order):

- Calculates all the wavelet coefficients of the N points signal up to nlevel. The coefficients are stored in a decomposition tree.

- Initialize the best basis with the nodes of the decomposition tree at the deepest level.

- Apply the merging criterion from the leafs to the root, using the criterion 'If two adjacent frequency bands have Gaussian coefficients, then merge the bands, otherwise let them be separated'. This operation leads to selecting the best decomposition basis which retains some nodes in the tree. The panel $\mathrm{C}$ gives a time-frequency representation of the best decomposition according to the kurtosis criterion. The spectrogram is given in panel B for comparison.

- Keep the frequency bands which are nonGaussian, i.e. characterized by an estimated kurtosis value less than the fixed threshold $\frac{1}{\sqrt{1-\alpha \%}} \cdot \sqrt{2^{\text {nlevel }} \frac{24}{N}}$. The coefficients in the other Gaussian bands are set to zero.

- Reconstruct the $\mathrm{N}$ points temporal signal.

- Apply a 'standard' transient detector, for instance an adaptive energy detector estimated at each instant $k$ by $\widehat{e_{2, k}}=\widehat{e_{2, k-1}}-\mu\left(\widehat{e_{2, k-1}}-x_{k}^{2}\right)$. The adaptive step $\mu$ controls the convergence rate.

Notice that the low frequency areas which are highly energetic have been merged in a single large frequency band because of the Gaussian nature of the noise in this frequency zone. On the other hand, the interesting region is greatly enhanced by an adapted fine segmentation around the frequency pulses produced by the transient appearance.

The panel F clearly shows the detection inability of the adaptive energy on the raw signal. By contrast, the de-noising procedure leads to an obvious peak in the detection curve. 
This simple example shows quite good results compared with the classical adaptive energy detector. Other techniques based on HOS or WT are also available. We compare in section 3.3.2 the performances of the proposed hybrid detector with existing HOS or WT based detectors. In section 3.3.3, we point out the efficiency of our kurtosis criterion best basis search by comparing its performances with the other classical criteria. A study of performances as a function of the parameters has been carried out in the section hereafter. This study is realized on the four test transients.

\subsection{Comparison and performance}

The comparison between detection methods is made in terms of performance ROC curves (Receiver Operating Characteristics). Experimental studies of performances are carried out with Monte-Carlo simulations. The exact procedure is explained in [16].

\subsubsection{Choosing the parameters}

The purpose of this study is the following:

- To obtain suitable values for a correct detection.

- To know the parameters that may influence the quality of detection.

For a fixed SNR, we have considered for each 4096 samples test transient depicted in figure 6 a set of different values for the three following parameters:

- The depth of decomposition defining the finest possible bandwidth in the decomposition has been set to the different following values nlevel $=\{4,5,6\}$. Increasing the value of this parameter allows to more precisely select or discriminate the frequencies. On the other hand the decreasing number of samples leads to non sufficiently reliable estimations for correct decisions.

- The order of the Daubechies wavelet has been experimented to the values order $=\{2,8,16,32\}$. This value controls the number of vanishing moments of the Daubechies wavelet which is related to the regularity of the wavelet.

- The frequency band merging decision furnished by the kurtosis based criterion has been made according to three confidence percentage values $\{85 \%, 90 \%, 95 \%\}$. The confidence percentage influences the segmentation rate.

The results of the simulation are presented in the array given below. 


\begin{tabular}{|c|c|l|l|l|}
\cline { 2 - 4 }$($ Slevel=4 & nlevel=5 & nlevel=6 \\
\hline Spool & $85 \%$ & & & \\
& $90 \%$ & & order=8 & \\
\hline Bottle & $95 \%$ & & & \\
$($ SNR=-6dB $)$ & $85 \%$ & & & \\
& $90 \%$ & & & \\
Manhole cover shock & $85 \%$ & & & \\
$($ SNR=-6dB $)$ & $90 \%$ & & & order=32 $=32$ \\
& $95 \%$ & & & \\
\hline Impulsive shock & $85 \%$ & & & \\
$($ SNR=-2dB $)$ & $90 \%$ & & order=16 & \\
& $95 \%$ & & & \\
\hline
\end{tabular}

For clarity reasons, we only give the parameter values that produce the best results, for each test transient. The performance evaluations are made through experimental ROC curves comparisons.

Practically, the relative variations of the performances are rather small. The search for the best performances is not an obvious exercice and the results are certainly not highly significant.

Nevertheless, we notice that the value nevel $=5$ is the best one except for the manhole cover shock where nlevel $=6$ is better. For a $6 \mathrm{kHz}$ sampling frequency, this corresponds to a finest bandwidth equal to $94 \mathrm{~Hz}$. A good frequency resolution is indeed necessary to correctly discriminate the various frequencies present in the manhole cover shock as observed in the figure 6 .

Concerning the wavelet order, the effect of this parameter is almost insignificant on the quality of detection. Nevertheless, the order must be sufficiently high to whiten the noise spectrum. For an already white noise it has no effect since wavelets are orthonormal. When the noise is colored with a correlation function $\Gamma_{x}(t)$, a simple computation leads to:

$$
E\left[P O_{s f p} P O_{s f p^{\prime}}^{*}\right]=2^{s} \int\left(\Gamma_{x}\left(2^{s} t\right) \star \psi_{f}(t-p)\right) \cdot \psi_{f}\left(t-p^{\prime}\right) d t
$$

As $s$ (i.e. the decomposition level) increases, the correlation function narrows and tends to act as a neutral element in the convolution. The expression restricts to the Dirac function $\delta\left(p-p^{\prime}\right)$. In the same manner, when the regularity of $\psi_{f}$ is increased by choosing higher orders of the Daubechies wavelet, the correlation function has less effect on the convolution product because the wavelet support enlarges with the wavelet order.

In [15], Pastor \& Gay have theoretically proved that the wavelet coefficient sequences tend to be whitened with the decomposition level and the filter regularity. More precisely, they have shown that the whitening was not the same for all the wavelet packets because the second order of the coefficients which is a whitening measure depends on the frequency localisation of the wavelet packets. 
For the greatest part of the experimented transients, the most suitable confidence percentage value seems to be $90 \%$. For the bottle transient, the results are better if the confidence percentage value reaches $95 \%$. The bottle presents peaks in high frequencies which are located in a flat area of the noise spectrum. The danger is to segment the low frequencies bands too much because of the important energy fluctuations in this area. A higher confidence percentage favours a frequency band merging and is more appropriate.

A brief comparison with other detectors is proposed in the next section: The performance are first exposed for a single HOS based method and in a second time for a WT based method.

\subsubsection{Comparison with HOS and WT}

Measuring a distance from Gaussianity An interesting detector proposed by Hinich in $[11,10]$ aims to detect a deviation from Gaussianity by a bispectral measure on temporal blocks. The bispectrum is a third-order statistic (2D Fourier transform of the tricorrelation) which theoretically equals to zero. The test evaluates the energy of the bispectrum modulus estimated in its principal domain on a temporal bloc. For a nonGaussian stationary signal, the test should equal to zero. Another test estimates the bispectrum in an area which is outside its principal domain. Its values equal to zero only if the signal is non-stationary and nonGaussian. In this case, a correct estimation of the bispectrum needs the signal to be sampled at least three times its highest frequency. Otherwise the test may not be correct because it can not discern bispectrum samples due to non-stationarity from samples due to overlapping.

We have evaluated the performances of the two real transient signals 'manhole cover shock' and 'impulsive shock' embedded in real noise respectively at $-6 \mathrm{~dB}$ and $-2 \mathrm{~dB}$ (figure 9). The ROC curves show that the hybrid approach HOS-WT performs better than the bispectral detector for these two transients. Indeed, for small probability of false alarm (say $2 \%$ ) gains of about $15 \%$ are reached in probability of detection.

The bispectrum method does not intrinsically realize a local time and frequency analysis since the test is evaluated on an entire temporal block and a set of bifrequencies defined in a global area.

Local time-frequency filtering The second approach studied relies on analysis tools specific for non-stationary signals: The idea is to calculate a time frequency representation and to estimate a test on this representation. In this sense, Frisch \& Messer in [8] have proposed a detector designed like a matched filtering in the wavelet transform domain. They use a set of transient models. The Generalized Likelihood Ratio Test derived gives a measure of ressemblance by locally comparing the signal with each model of the library. The comparison is made with a scalar product between the 4 wavelet coefficients patterns of the signal and the model over the time-scale plane. The pattern represents a local time scale area in the representation. The scalar product is high when the patterns are fitting.

Like classical matched filtering, the method gives very good results as long as the functions in the library are 'near' from the transient. The method is not robust and cannot be employed because it would restrict the study to some specific kinds of transients.

Other HOS or WT methods obviously exist but picking up a few of them show that considering 
the tools independently is not optimal. Wavelet packets allow to merge both approaches and to search for example the most nonGaussian basis. We analyse in the next section the performance of the de-noising algorithm when classical best basis search criteria are used.

\subsubsection{What about the other classical best basis search procedures?}

Classical best basis search procedures rely on a criterion whose purpose is to minimize a basis construction cost. The approach proposed in this article is quite different because the criterion is built with a detection goal. What is at stake is to decide whether or not samples are Gaussian and to keep nonGaussian coefficients. This detection formulation naturally leads to an automatic de-noising threshold.

None of the existing cost functions have been used for detection. Indeed initial purpose was compression involving an information measure realized by entropy, logarithmic energy or $l^{p}$ norm. Instead of minimizing a cost functional allowing to get the 'cheapest' basis in terms of coding, one can imagine to search for the 'most nonGaussian' basis [4]. This can be done by choosing the basis which maximizes the kurtosis of the coefficients.

At this point, the proposed thresholding value can be used to get rid of the Gaussian frequency bands in the obtained time-frequency tiling. Experiments show that results are as good as the proposed detection method except for very low SNR. For frequency multi-component transients, the detection driven basis search method gives better results (figure 10).

After performance and comparison results, we propose a real case study encountered in an industrial context. The signals presented and analyzed for this illustration have been furnished by the French National Electricity Company that we gratefully acknowledge.

\section{Application to missing bolt detection in nuclear plants}

The first application depicted in paragraph 3.2 allowed to validate the algorithm through simulations and performance curves. Experiments were controlled by embedding real transients in recorded sea noise. Beyond these simulated situations we tested the method on a real problem of detection appearing in nuclear plant pipings. Sensors placed on the pipes sometimes detect hits in the inner sides of pipes produced by any forgotten or detached object. For example the wandering bodies detected in this application has been identified as screw bolts which have been forgotten after pipe cleaning operations. Actually, such problems occasionally appear in nuclear plants and are of great importance. Indeed the wandering bodies can cause damage which requires repair and necessitates shutting the plant down. This produces important costs thus requiring most correct detections as possible.

The existing method based on a spectral substraction suffers from a too significant false alarm rate at the fixed detection rate. The reason is the following: Energy fluctuations are detected as they should not. The fluid circulating in the pipes produces hydraulic noise which is actually subject to some energy fluctuations along the time. The proposed detection method essentially takes into account the statistical properties of the noise along the time. In the present case, the hydraulic noise looks Gaussian and stays Gaussian whatever its power may be. 
A wandering bodies signal is presented figure 11 (panel A). The signal is sampled at a rate of $50 \mathrm{kHz}$. The spectrogram of the signal appears in panel B. It is calculated on 256 points segments with $50 \%$ overlapping. Segments are weighted with Hanning windows. Transient events can clearly be seen in the signal around 1 second. Four narrow bands can be seen in the spectrogram (at about $3,6,10$ and $11 \mathrm{kHz}$ ). These bands correspond to hydraulic noise due to the fluid. In some particular working conditions, fluid circulating generates frequency bands in the pipes acting as a wave guide. The $3 \mathrm{kHz}$ frequency band is the most powerful and its energy fluctuates a lot along the time. This hides the presence of transients which are difficult to be seen in the time domain. By contrast, the most powerful transients are clearly depicted by the spectrogram. However, we can wonder if other screw bolts signatures are present in the signal.

The time frequency representation obtained with the described algorithm is shown in panel C. The decomposition depth is set to 6 (i.e. 64 frequency channels). Bands have been merged according to their coefficients Gaussianity with a $90 \%$ confidence made decision. A sufficient regular wavelet has been chosen such as a Daubechies one constructed on a 16-taps filter. The same representation after thresholding is represented in panel $\mathrm{D}$. The kurtosis curve plotted at the right have permitted to retain the nonGaussian frequency bands. It is very interesting to see that the powerful frequency bands at 3, 10 and $11 \mathrm{kHz}$ have been considered as Gaussian by the algorithm and then have been eliminated. The reconstructed signal is shown in panel E. An adaptive detector is then applied on that signal. It is shown in panel $\mathrm{F}$ where we also plot the adaptive energy before any processing for comparison (signal have been normalized in energy). We can see that the false alarm before processing have been eliminated, at the instant 0.1 second. The false alarm may be due to the high amplitude of the $3 \mathrm{kHz}$ band at this instant. Note also the spectacular gain obtained in the detection, by contrast between the curves. Furthermore, a transient at 1.1 second is detected by the hybrid detector whereas it is not seen by the energy on the raw signal. The proposed detector on this example decreases the false alarm rate and reveals a miss detection not solved by the energy detector.

\section{Conclusion}

In this paper we use an adaptive time-frequency plane segmentation for a transient detection problem. We take advantage of the segmentation flexibility proposed by the wavelet packets theory to find the 'most adapted' basis among a library of possible wavelet packet bases.

The segmentation process is guided by a detection purpose: Trying to make transients emerge from the noise in the time-frequency representation. The discrimination is based on the knowledge of the signals we study: The wavelet coefficients of the transient signals are nonGaussian whereas the coefficients of the noise are Gaussian. The derived criterion merges Gaussian frequency bands. The simulation results in terms of ROC curves show that the combination of HOS tools with timefrequency representations is efficient. They also show the importance of the time-frequency plane segmentation in the transient detection problem.

Let us recall the key-parameters in the proposed algorithm. The results essentially depend on the confidence value attributed to the decision criterion. It defines the segmentation tendency by facilitating the merging or not. Above all, the de-noising procedure used is particularly interesting because the threshold is automatically fixed by the confidence value and the chosen decomposition depth. Finally the algorithm can be considered as an automatic band-pass filtering. 
Beyond the beginning applications considered in passive sonar, we have shown interesting results appearing in an industrial context. The algorithm can be used both as a detection method and as a de-noising method. In the wandering bodies problem, the main objective is to make clear disturbing situations. However the other de-noising application is also very useful for an event classification. The classification allows to identify the cause of the detected hits and then to evaluate the seriousness of the situation.

Other applications may be considered. For example radio astronomy recordings are perturbed by powerful interferences stemming from satellite communications. Some of the perturbations can be modeled as unknown pure frequencies. The current methods detect the appearance of such interferences and then stop the recordings - the method is named time-blanking. Our detector could detect the disturbing frequency bands which are nonGaussian. On the contrary Gaussian components would be kept in the de-noising procedure so that radio astronomic recordings would not stop.

Beside other new applications, future works concern the extension of the algorithm to a 'double tree' method: It allows to select the best basis among a set of best bases found when the initial signal is time sliced [9]. A binary primary tree is build. It segments the initial signal up to a certain level in the time domain. On each node, a wavelet packet decomposition tree is developed in which a best basis is selected. Each node of the primary tree is then affected with its best basis construction cost. The final best basis is selected with the search algorithm in the primary tree. This method needs yet to be adapted for a detection purpose.

\section{References}

[1] P. Abry and P. Flandrin. Multiresolution Transient Detection. In IEEE-SP International Symposium on Time-Frequency and Time-Scale Analysis, pages 225-228, Philadelphia, USA, Octobre 1994.

[2] P.-O. Amblard and Ph. Ravier. Experimental performance analysis of an on-line transient detector. In IEEE Signal Processing Workshop on Higher-Order Statistics, pages 171-175, Barcelona, Spain, 1995.

[3] P. L. Brockett, M. J. Hinich, and G. R. Wilson. Nonlinear and non-gaussian ocean noise. Journal of Acoustical Society of America, 82(4):1386-1394, October 1987.

[4] J.B. Buckheit and D.L. Donoho. Time-frequency tilings which best expose the non-Gaussian behavior of a stochastic process. In IEEE-SP International Symposium on Time-Frequency and Time-Scale Analysis, pages 1-4, Paris, France, June 1996.

[5] G. Carrault, L. Senhadji, J. Bellanger, G. Passariello, and F. Mora. Analyse et représentation par transformée en ondelettes d'un signal ECG. In Colloque sur le Traitement du Signal et des Images, GRETSI, pages 141-144, Juan-Les-Pins, France, 1991.

[6] L. Duboisset-Chareyre. Analyse bispectrale de signaux réels. Application à la détection de signaux transitoires. PhD thesis, INP Grenoble, 1997.

[7] B. Friedlander and B. Porat. Performance Analysis of Transient Detectors Based on a Class of Linear Data Transforms. IEEE Transactions on Information Theory, 38(2):665-673, 1992.

[8] M. Frisch and H. Messer. The use of the Wavelet Transform in the Detection of an Unknown Transient Signal. IEEE Transactions on Information Theory, 38(2):892-897, March 1992.

[9] C. Herley, J. Kovačević, K. Ramchandran, and M. Vetterli. Tilings of the time-frequency plane: Construction of arbitrary orthogonal bases and fast tiling algorithms. IEEE Transactions on Signal Processing, 41(12):3341-3359, December 1993. 
[10] M. J. Hinich. Detecting a transient signal by bispectral analysis. IEEE Transactions on Acoustics, Speech and Signal Processing, 38(7):1277-1283, July 1990.

[11] M. J. Hinich and G. R. Wilson. Detection of non-gaussian signals in non-gaussian noise using the bispectrum. IEEE Transactions on Acoustics, Speech and Signal Processing, 38(7):1126-1131, July 1990.

[12] M. G. Kendall and A. Stuart. The advanced theory of statistics, distribution theory. Charles Griffin \& Company Limited, 1977.

[13] J.-L. Lacoume, P.-O. Amblard, and P. Comon. Statistiques d'Ordre Supérieur pour le Traitement du Signal. Masson, 1997.

[14] S. G. Mallat. A theory for multiresolution signal decomposition: the wavelet decomposition. IEEE Transactions on Pattern Analysis and Machine Intelligence, 11(7):674-693, July 1989.

[15] D. Pastor and R. Gay. Décomposition d'un processus stationnaire du second ordre. Propriétés statistiques d'ordre 2 des coefficients d'ondelettes et localisation fréquentielle des paquets d'ondelettes. Traitement du Signal, 12(5):393-420, 1995.

[16] P. Ravier and P.-O. Amblard. Combining an adapted wavelet transform with 4th order statistics for transient detection. Signal Processing, 70(2):115-128, 1998.

[17] Ph. Ravier. Détection de transitoires par ondelettes adaptées - Critères d'adaptation fondés sur les statistiques d'ordre supérieur -. PhD thesis, INP Grenoble, 1998.

[18] L. Senhadji, G. Carrault, and J. Bellanger. Détection de transitoires épileptiques dans le signal EEG par transformée en ondelettes. In Colloque Temps-Fréquence, Ondelettes et Multirésolution, INSA-Lyon, pages 22.1-22.4, 1994.

[19] L. Senhadji, G. Carrault, and J. Bellanger. Interictal EEG Spike Detection: A New Framework Based on Wavelet Transform. In IEEE-SP International Symposium on Time-Frequency and Time-Scale Analysis, pages 548-551, Philadelphia, USA, Octobre 1994.

[20] M. V. Wickerhauser. Adapted wavelet analysis from theory to software. A.K. Peters Wellesley, Massachussetts, 1994. 
(a)

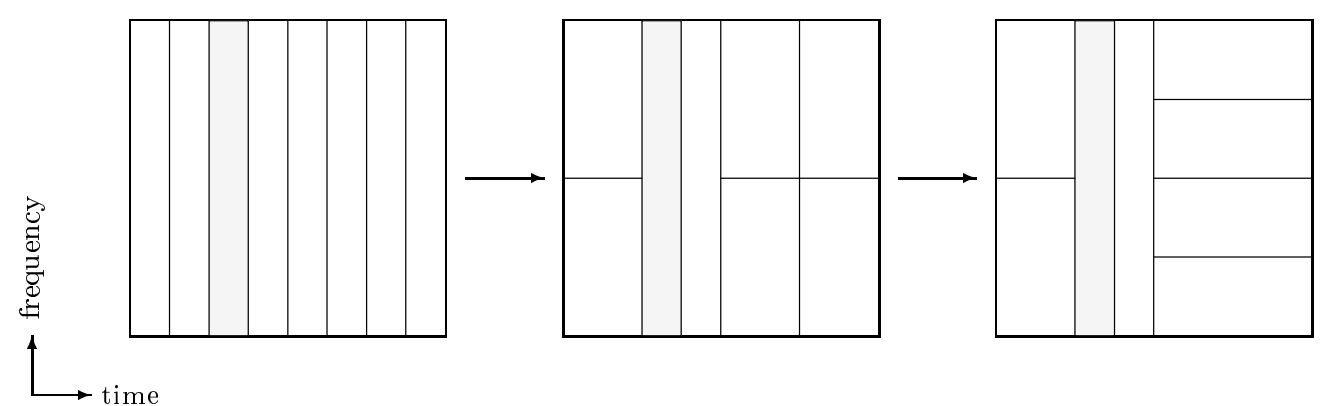

(b)

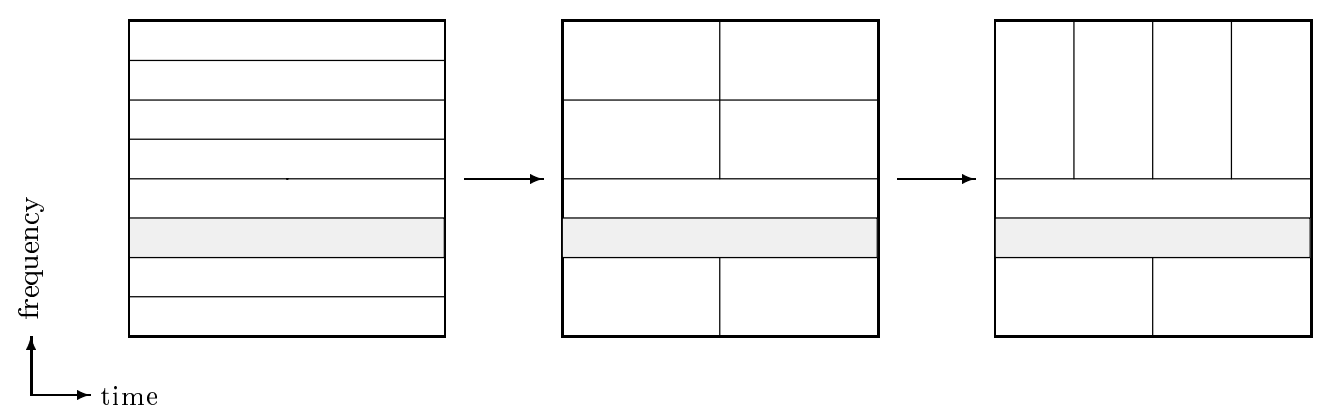

Fig. 1. (a) Searching for the best basis using Malvar wavelets - (b) Searching for the best basis using wavelet packets

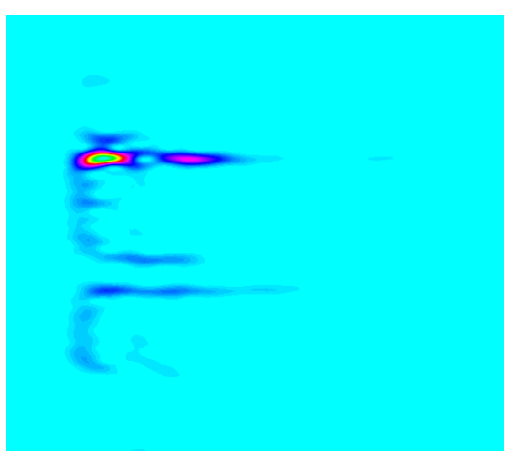

(a)

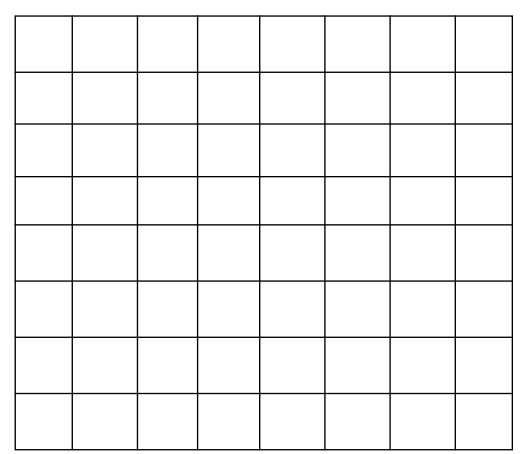

(b)

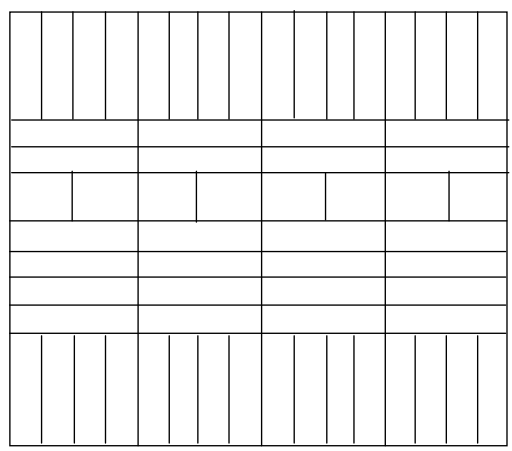

(c)

Fig. 2. (a) Continuous short time Fourier representation - (b) Windowed Fourier basis - (c) Adapted wavelet packet basis.

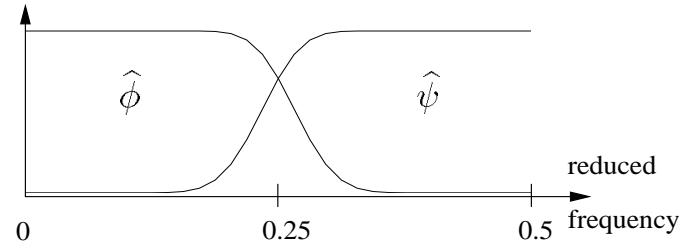

(a)

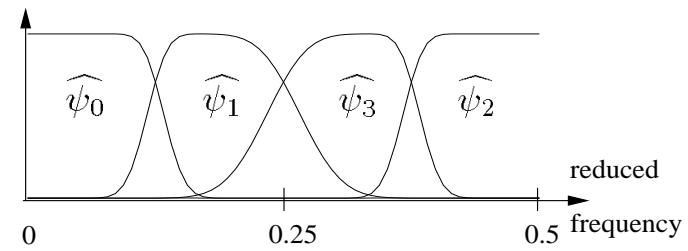

(b)

Fig. 3. (a) High-pass filter $\widehat{\psi}$ and low-pass filter $\widehat{\phi}$ used in wavelet transform - (b) Band-pass filters $\widehat{\psi_{0}}, \ldots, \widehat{\psi_{3}}$ used in wavelet packet transform. A four spectral Daubechies16 wavelet family is represented. 

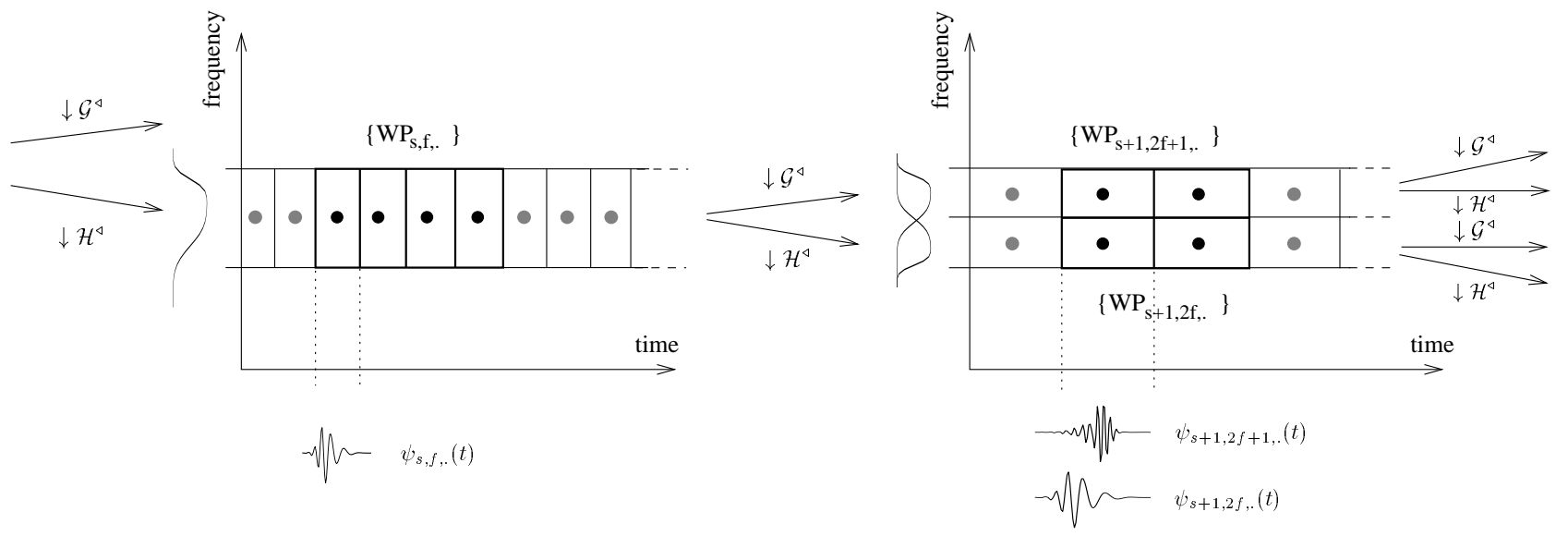

Fig. 4. Application effects of the operators $\downarrow \mathcal{H}^{\triangleleft}$ and $\downarrow \mathcal{G}^{\triangleleft}$ on the time-frequency tiling.
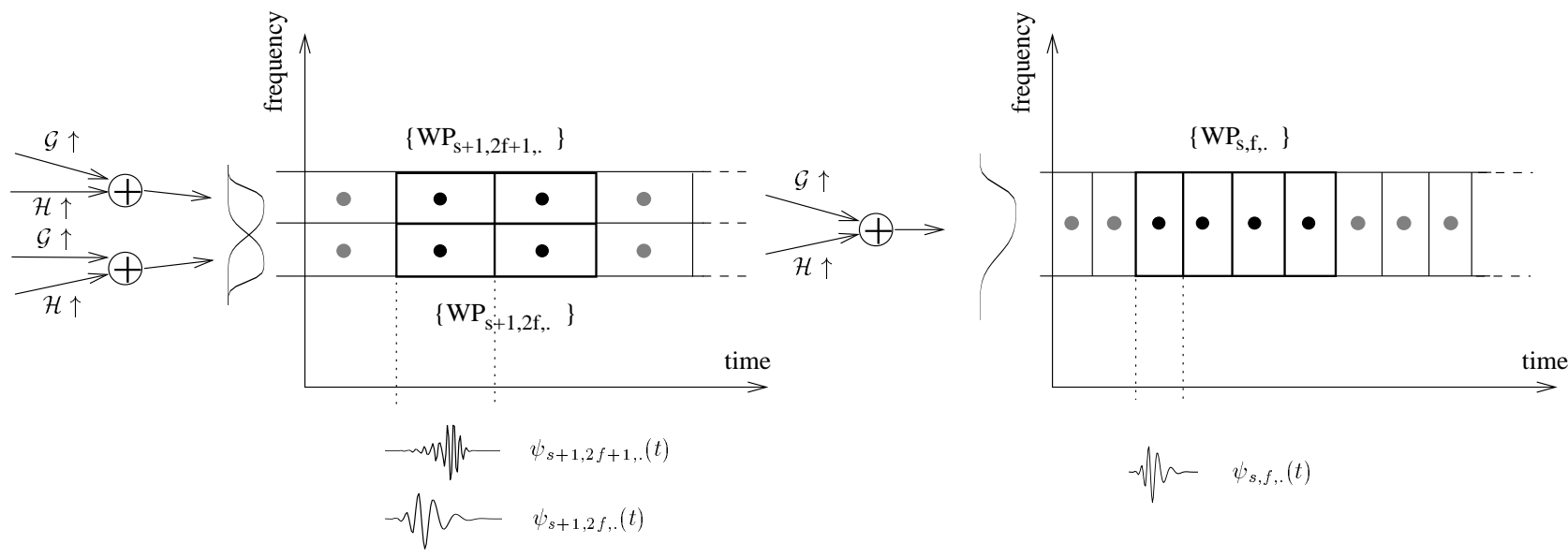

Fig. 5. Reconstruction of the coefficients $W P_{s, f, p}$ by applying the filters $\mathcal{H} \uparrow$ and $\mathcal{G} \uparrow$ on the initial coefficients. The induced modified time-frequency tiling is represented. 

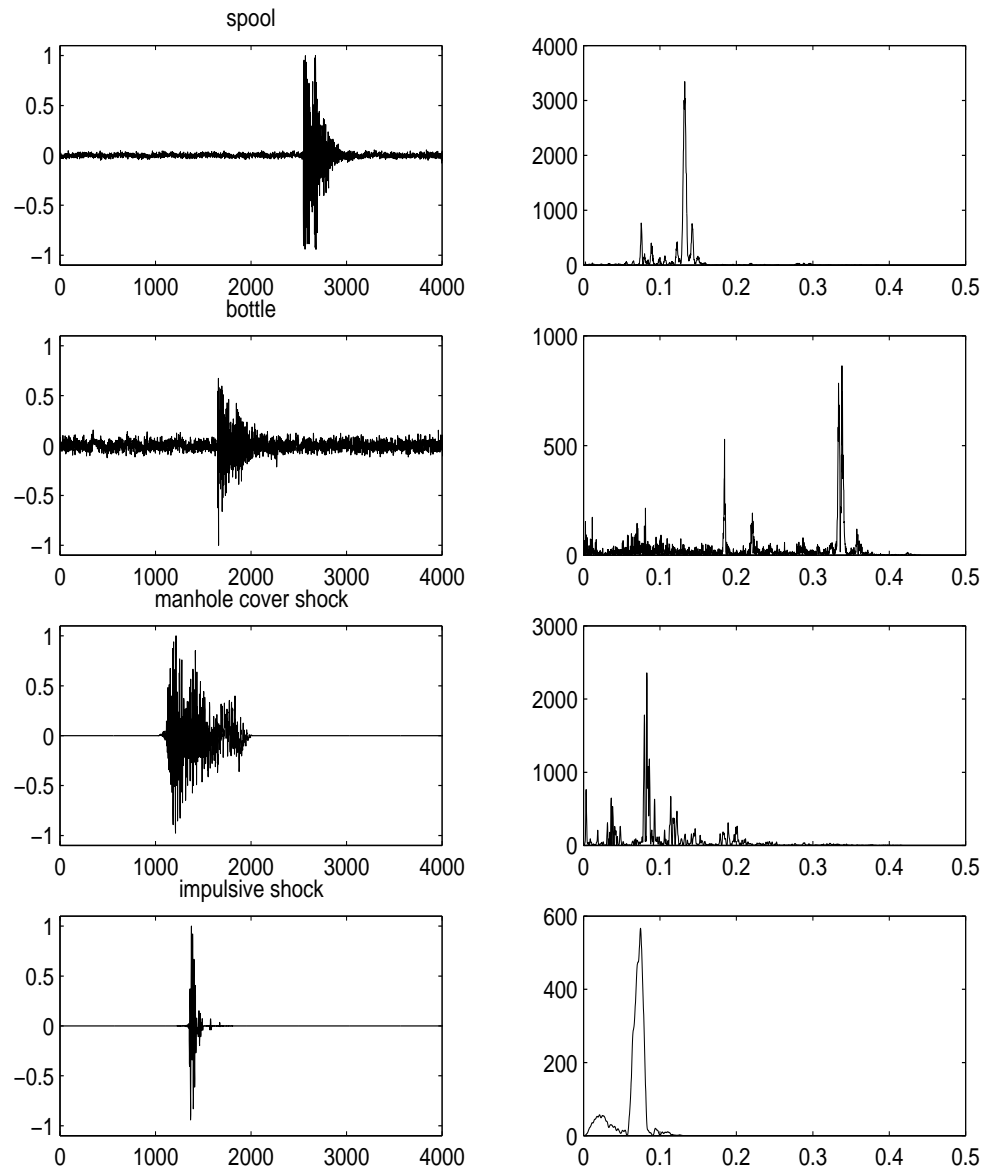

Fig. 6. Transients used for the experiment. Temporal shape (left) and spectral shape (right). Sample number in time and reduced frequencies are represented in abscissa.
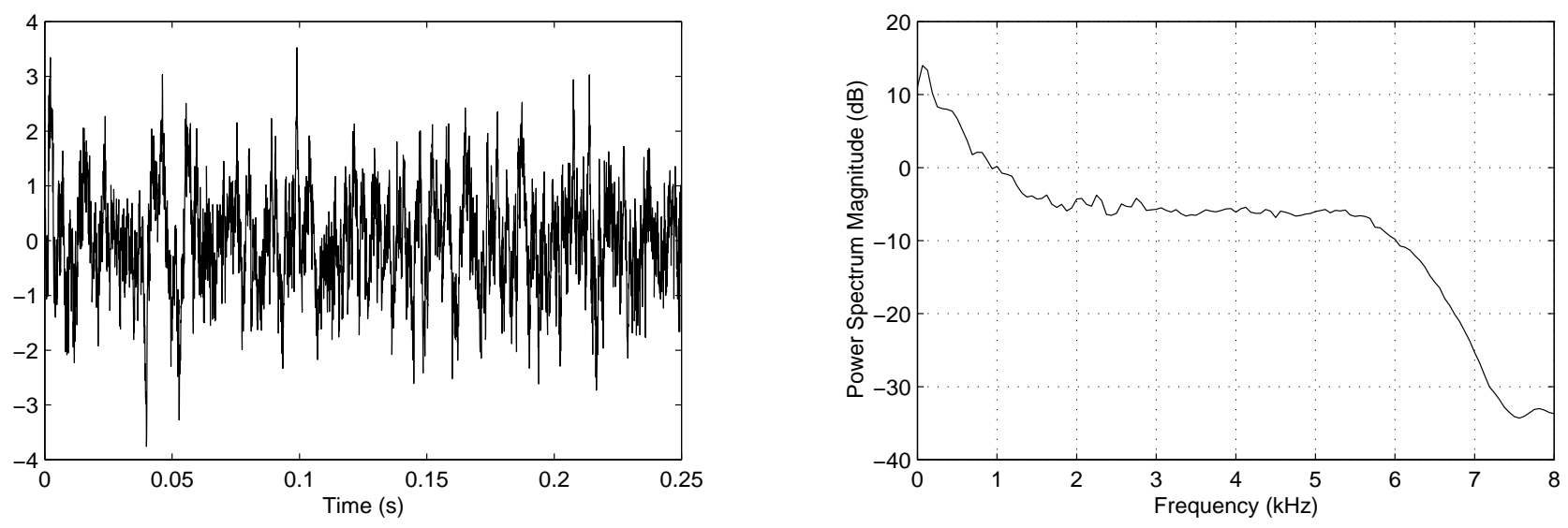

Fig. 7. Temporal shape (left) of a 4000 points sea noise realization. Power spectral density shape (right) of the entire sea noise recording (70000 points). The PSD is estimated on Hanning windowed 256 points slices. 

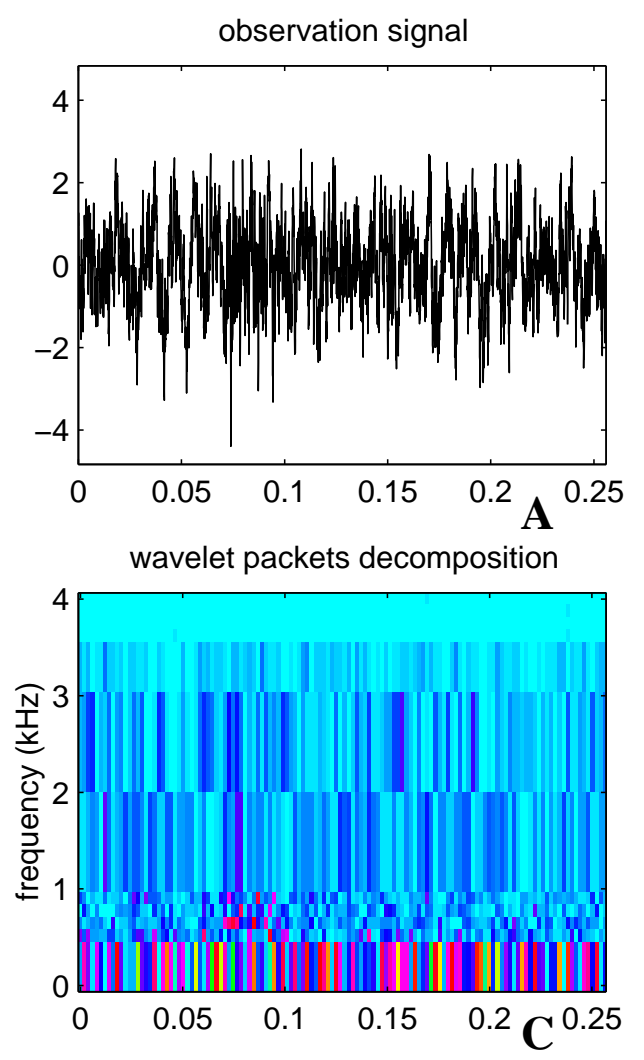

signal after thresholding

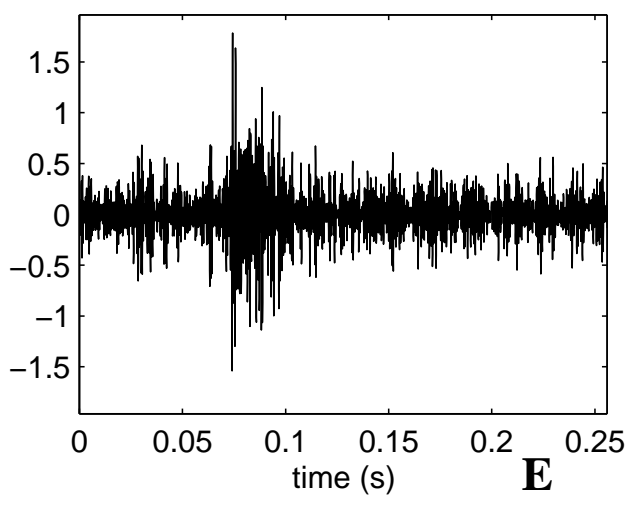

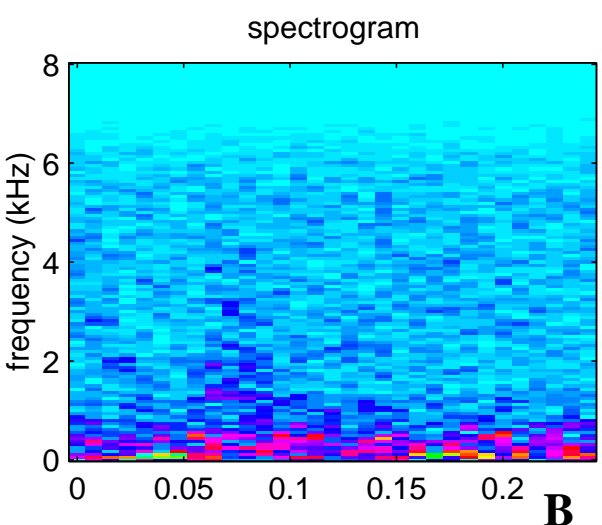

denoising by coefficients thresholding
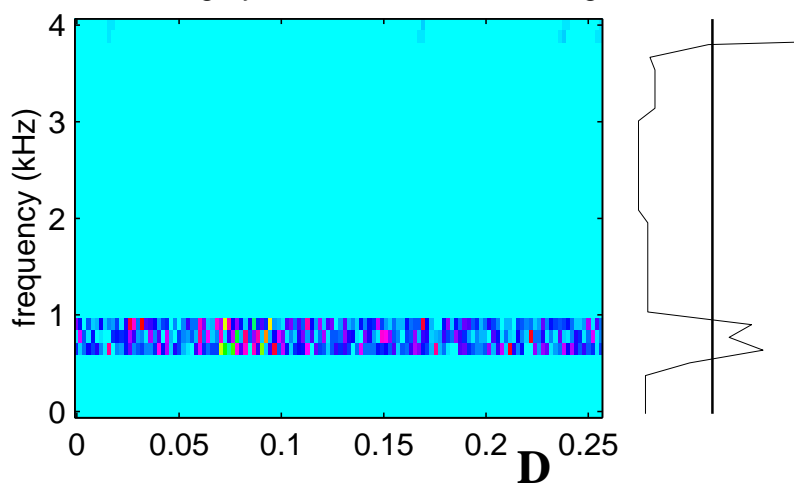

Fig. 8. Detection of the transient 'manhole cover shock' embedded in the sea noise at $-6 \mathrm{~dB}$. A: signal, B: spectrogram, C: time-frequency representation obtained by the best wavelet packet basis, D: idem after thresholding the Gaussian coefficients, E: reconstructed signal, F: adaptive energy before and after thresholding. 

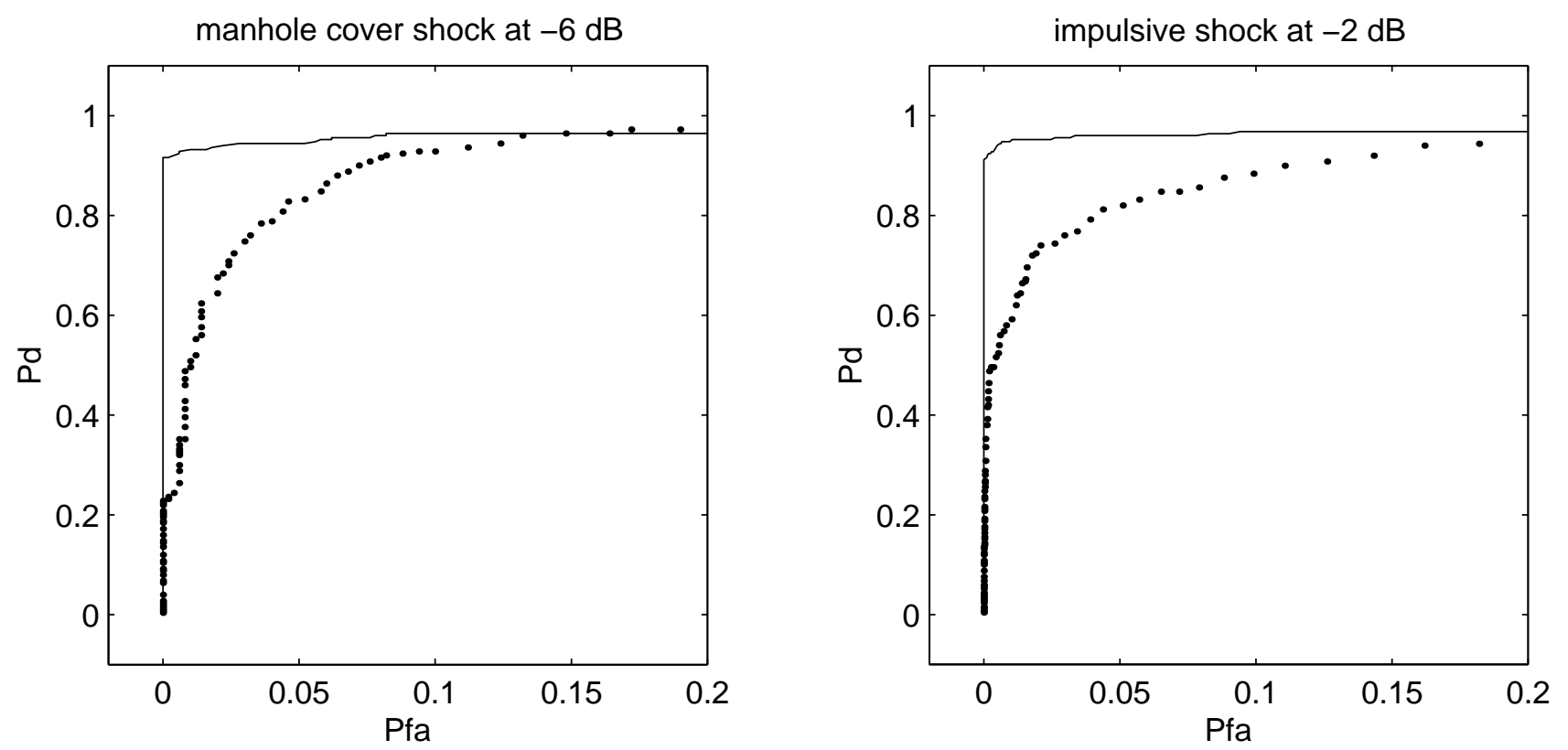

Fig. 9. ROC curves for transient 'manhole cover shock' at $-6 \mathrm{~dB}$ (left) and transient 'impulsive shock' at -2 $\mathrm{dB}$ (right). - proposed detector, ... bispectral detector.

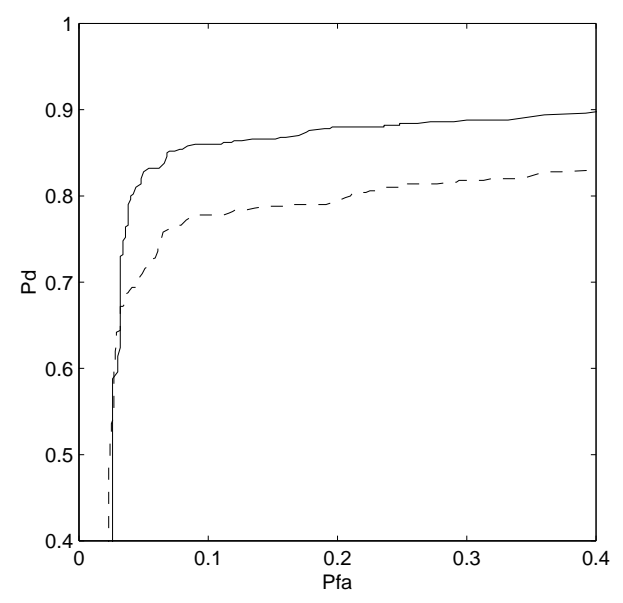

Fig. 10. Comparison between the detection driven search $(-)$ and the optimizing cost driven search (-) at very low SNR (-8 dB). A gain up to $10 \%$ in probability of detection is reachable for the manhole cover transient detection with the appropriate method. The curve is plotted in the interesting false alarm probability region. 

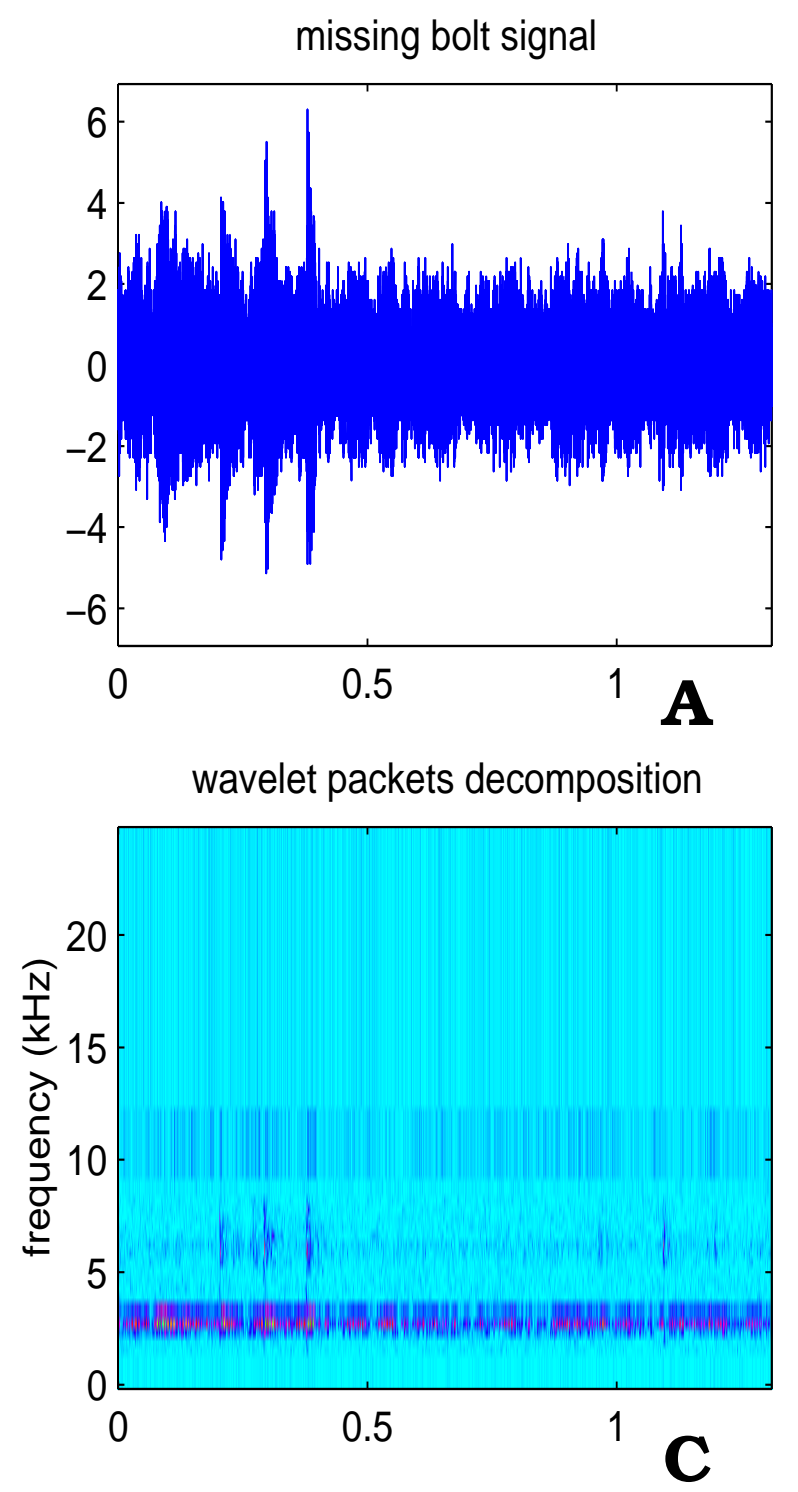

signal after thresholding

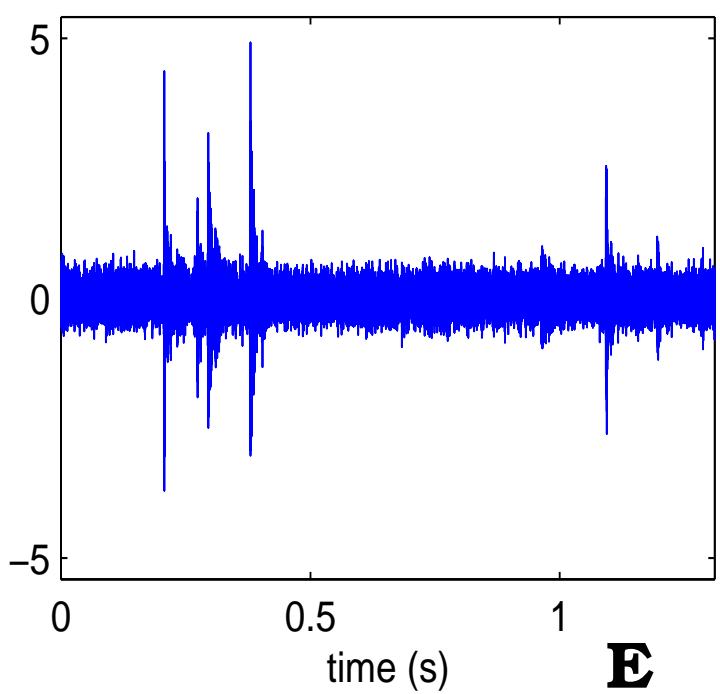

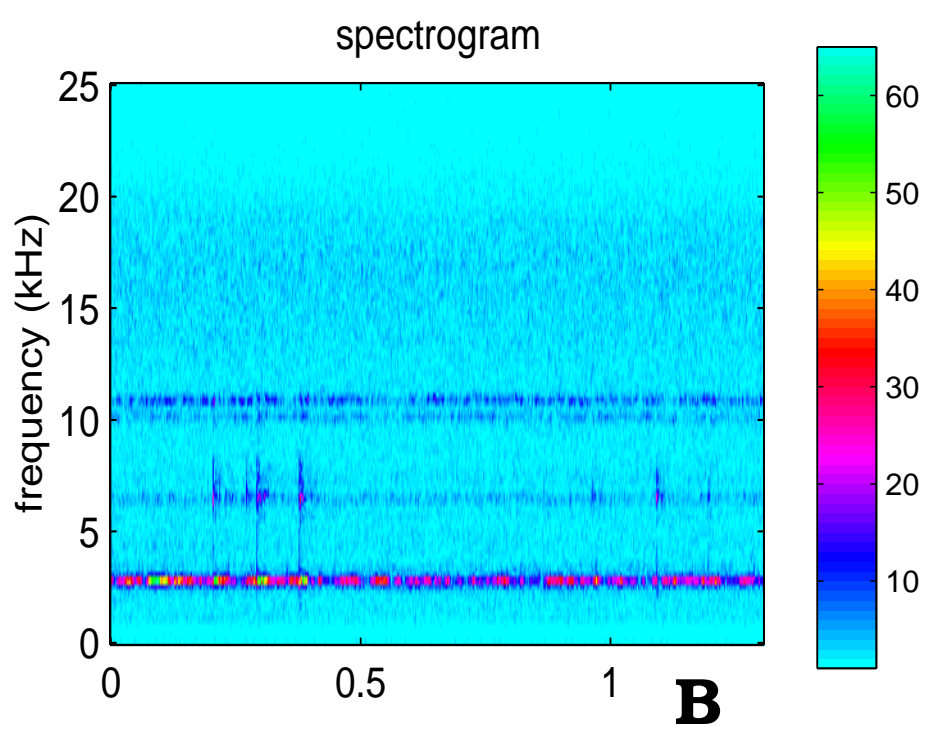

denoising by coefficients thresholding

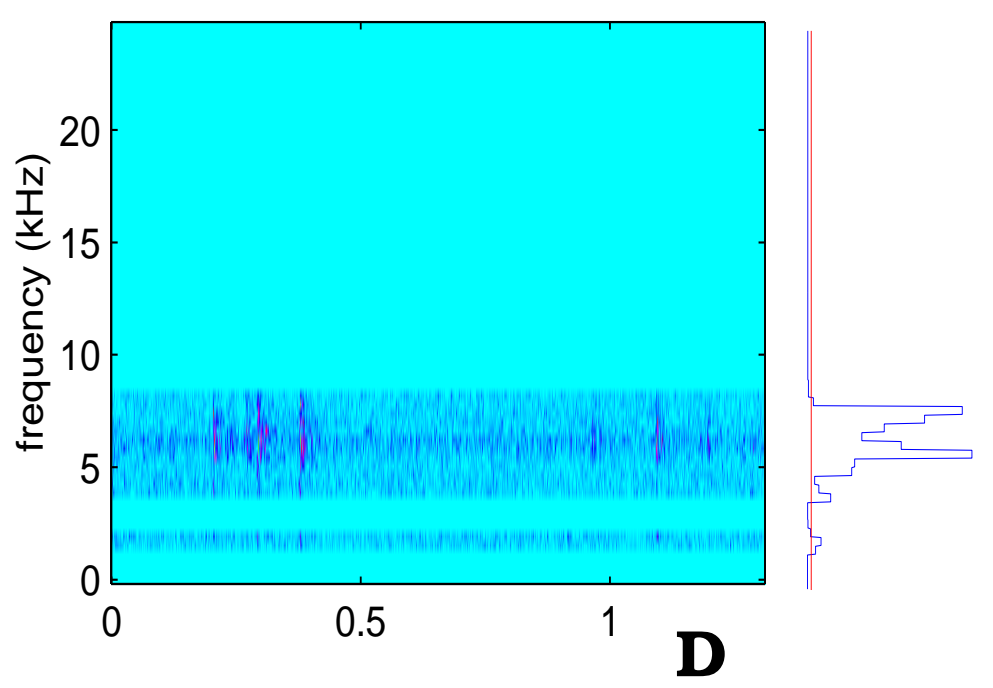

adaptative energy detectors

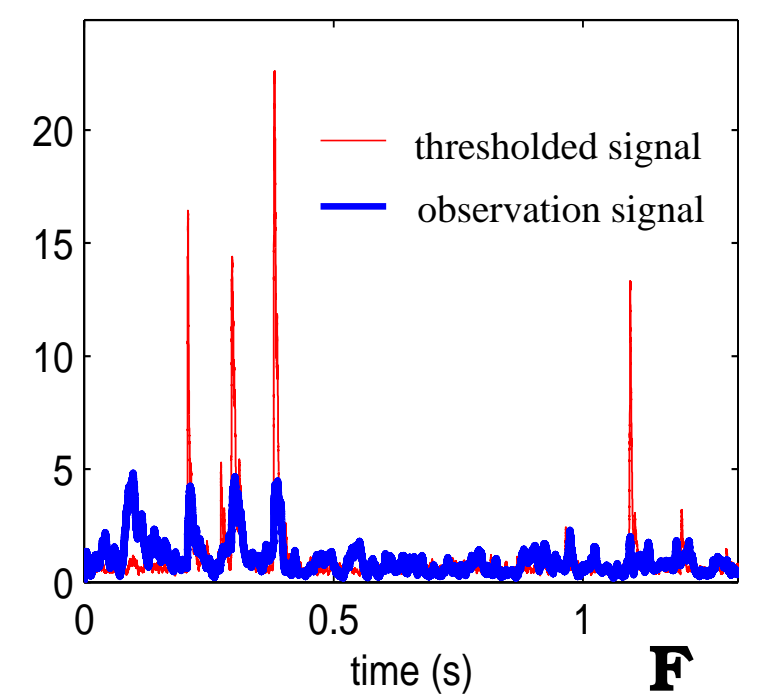

Fig. 11. Detection of forgotten screw bolts in the piping of nuclear plants. A: Signal, B: Spectrogram, C: Time-frequency representation obtained by the best wavelet basis, D: Idem after thresholding of Gaussian coefficients, E: Reconstructed signal, F: Adaptive energy before and after thresholding. 